the $10^{\text {th }}$ centile. Ten women had bipolar disease and 6 used antipsychotics. One $(16 \%)$ baby was below the $10^{\text {th }}$ percentile.

In total 12 women took olanzepine and of these 8 (66\%) babies had a congenital anomaly, 4 had tongue tie but 3 babies (on fluoxetine and olanzepine) had major congenital heart disease.

Conclusion Women referred to a mental health clinic had increased rates of growth restriction compared with the general population. Numbers are small but association of antenatal anti-psychotics with fetal growth restriction and congenital anomalies needs to be considered.

\section{PM.66 WITHDRAWN BY AUTHOR}

\section{PM.67 BOWEL PERFORATION FOLLOWING SEVERE CONSTIPATION IN PREGNANCY: A CASE REPORT}

doi:10.1136/archdischild-2013-303966.148

SJ Bonner, J Ford, F Soydemir. Royal Preston Hospital, Lancashire, North West, UK

Stercoral perforation is defined as "perforation of the bowel due to pressure from a faecal mass." This is due to an accumulation of stool that has hardened and has remained in the bowel over a long period of time causing stagnation and deformity of the large intestine.

At 25 weeks gestation a lady attended with severe constipation despite various stimulants/enemas and left sided abdominal pain. She started vomiting and felt generally unwell. She had abdominal distension and sluggish bowel sounds. Haematology and biochemistry were normal. Ultrasound showed large amounts of gas in the bowel but was otherwise unremarkable. The surgeons reviewed her, excluded any obstruction and continued conservative management with observation and laxatives. Her pain and constipation continued until she became tachycardic and tachyopneic, her CRP began to rise. Prophylactic steroids were given. Antibiotics were commenced and urgent MRI arranged. MRI showed no obvious stricture or structural obstruction. A Naso-gastric tube was passed draining bilious fluid. Throughout the day her clinical condition deteriorated. She was transferred to delivery for critical care review and surgical input.

She eventually went to theatre for a de-functioning iliostomy. At laparotomy bowel perforation was identified with faecal peritonitis. She had a colectomy. Due to maternal condition and access and post operative considerations the baby was delivered by caesarean section.

Although constipation is common amongst pregnant women it is usually corrected by conservative measures such as stimulants/ enemas and adequate hydration. Refractory cases should prompt early referral for investigation for underlying pathology.

\section{PM.68 MANAGEMENT DILEMMAS OF CONN'S SYNDROME IN PREGNANCY}

doi:10.1136/archdischild-2013-303966.149

JE Ford, SJ Bonner, S Gupta. Royal Preston Hospital, Preston, North West, Lancashire, UK

The most common cause of primary hyperaldosteronism is Conn's syndrome $(80 \%)$, and treatment resistant hypertension with hypokalaemia in pregnancy should prompt investigation for this. There is physiological up-regulation of the rennin-angiotensin system during pregnancy, resulting in secondary hyperaldosteronism. The true incidence of Conn's syndrome in pregnancy is unknown but low, and vague presentations make diagnosis and management a dilemma.

A 25 year old primigravida presented at 26 weeks with hypertension, proteinuiria and bilateral leg oedema. She had an emergency caesarean section for severe pre eclampsia. Persistent hypokalaemia lead to a subsequent diagnosis of Conn's syndrome. She had normal adrenal imaging. She began pharmacological treatment with spironolactone. Currently she is 24/40 into her second pregnancy.

Spironolactone is contraindicated due to its teratogenic effects particularly virilisation of male fetuses. She has been commenced on low dose Aspirin and oral potassium supplements. Her renal function and Magnesium levels, along with blood pressure and urinalysis are being monitored regularly.

Studies have shown unsatisfactory hypotensive effects with methyldopa, hydralazine, labetolol, diazoxide and nifedipine. There is limited research available for eplenerone which is structurally similar to spironolactone but weaker and so does not have virilising effects, this is however unlicensed. Although the presentation of pathology can be non specific and "normal" in early pregnancy, treatment resistant hypertension requires assessment for primary hyperaldosteronism. Management has considerable difficulties due to the teratogenicity/lack of evidence on first line pharmacological treatments. So far there is supportive evidence that amiloride is safe and effective and could be used in these cases.

\section{PM.69 HAEMATINIC EVALUATION AND SUPPLEMENTATION IN A NORMAL PREGNANCY}

doi:10.1136/archdischild-2013-303966.150

${ }^{1} \mathrm{CM}$ McCarthy, ${ }^{2} \mathrm{MR}$ Cahill, ' $\mathrm{K}$ O'Donoghue. 'Department of Obstetrics and Gynaecology, Cork University Maternity Hospital, Cork, Ireland; ' 2 Department of Haematology, Cork University Hospital, Cork, Ireland

Maternal and Neonatal complications have been described in antenatal iron, folate and $\mathrm{B} 12$ deficiencies. ${ }^{1}$ International recommendations have described the investigation and treatment of haematinic deficiencies. ${ }^{2}$

A prospective audit was conducted between January and April 2012. Postnatal patient charts were randomly sampled. Data was entered into a secure database. Haematinic levels were retrospectively acquired using institutional laboratory systems.

176 patients were included in our sample group, with a total of 757 investigations. 155 patients had more than 2 sets of haematological investigations during pregnancy. $39.7 \%$ had haematinic investigations performed. 48 patients had a ferritin level below $30 \mathrm{ug} / \mathrm{L}$, of which 11 were using iron supplementation. $69.8 \%$ of patients reported folate supplementation, with $12.5 \%$ taking combined antenatal supplementation. $21 \%$ were taking a form of iron supplementation. Of the 28 people who were recorded as not taking antenatal supplementation, 6 had suboptimal ferritin levels.

Currently, there are no national guidelines on haematinic investigation in the antenatal population. This is imperative to improve patient outcomes. It is also essential to treat those who demonstrate clinical anaemia, and sub-optimal ferritin levels.

\section{REFERENCES}

1. Scholl, Hediger. Anemia and iron-deficiency anaemia: compilation of data on pregnancy outcome. Am J Clin Nutr 1994;59:492S-500S.

2. Pavord, Myers, Robinson, Allard, Strong, Oppenheimer. UK guidelines on the management of iron deficiency in Pregnancy. British Committee for Standards in Haematology. July 2011.

\section{PM.70 COMPARATIVE STUDY BETWEEN GESTATIONAL AND PREGESTATIONAL DIABETES IN RELATION TO GLYCEMIC CONTROL AS REGARDING FETAL AND NEONATAL OUTCOME}

doi:10.1136/archdischild-2013-303966.151

M Bahaa. Kasralainy Maternity Hospital, Cairo, Egypt

Background Pregnancy tends to reset the glucose homeostasis in the direction of diabetes. About $1-2 \%$ of all pregnant women 